\title{
Comparison of Frequency of Congenitally Missing Upper Lateral Incisors among Skeletal Class I, II and III Malocclusions
}

\author{
Nivedita Sahoo ${ }^{1}$, Rami Reddy ${ }^{2}$, Snigdha Gowd ${ }^{3}$, Bhagabati P Dash ${ }^{4}$, Jasbir Meher ${ }^{5}$
}

\begin{abstract}
Aim: The present investigation aims to find out the frequency of congenitally missing upper lateral incisors among skeletal class I, II, and III malocclusions and to find out its variability among different genders.

Materials and methods: The study sample included orthopantomograms (OPGs) and a lateral cephalogram of 1,000 patients retrieved from previous records. Of the selected samples, 290 were males and 710 were females. Orthopantomograms helped diagnose the presence of unilateral/bilateral maxillary lateral incisors.

Results and statistics: Of the 1,000 samples, 710 (71\%) were female and 290 (29\%) were male patients. An estimated 37 (3.77\%) patients were found to have missing maxillary lateral incisors. Of the female patients, $4.04 \%$ were having missing laterals, whereas $3.10 \%$ of the male patients were having agenesis of maxillary incisors. Totally, $0.9 \%$ of missing upper laterals were recorded in males, whereas $2.8 \%$ of missing lateral incisors in upper arch were found in females. A total of $54.16 \%$ of skeletal class I, $33.33 \%$ of skeletal class II, and $12.5 \%$ of skeletal class III cases were found to have missing upper lateral incisors.

Conclusion: The prevalence rate for congenitally missing upper lateral incisors in orthodontic adolescent Odia population in Bhubaneswar is $3.77 \%$. The females had a greater percentage of agenesis of the upper lateral incisor when compared to males. An estimated $54.16 \%$ of skeletal class I, $33.33 \%$ of skeletal class II, and $12.5 \%$ of skeletal class III cases were found to have missing upper lateral incisors. This difference might be due to genetic variations or different environmental conditions. A multidisciplinary treatment protocol must be planned to manage the missing maxillary lateral incisor and to restore the impaired esthetics and function.
\end{abstract}

Keywords: Lateral incisors, Space management, Tooth agenesis.

The Journal of Contemporary Dental Practice (2019): 10.5005/jp-journals-10024-2643

\section{INTRODUCTION}

Anodontia is the absence of teeth. Hypodontia means absence of less than six teeth. Agenesis is defined as teeth that were unsuccessful in developing at birth. Absence of the tooth causes alignment problems, arch length discrepancies, and dental asymmetry. ${ }^{1,2}$

That the formation and maturation of the teeth are strictly governed by factors such as genetics is a well-established theory. Few missing teeth in an individual are identified to be associated with several genetic and syndromic conditions. ${ }^{3-6}$ Mutations in MSX1, PAX9, and AXIN2 in families with multiple missing tooth ${ }^{7-9}$ have been proved by several studies in molecular genetics.

Moyers stated that there are five major recognized reasons for agenesis of teeth. He stated that heredity, syphilis, and rickets might predispose to agenesis. Some mutational and evolutionary changes in the dentition also lead to disturbances in formation of the tooth. ${ }^{10}$

Missing upper lateral incisors represents a clinical problem impairing dental esthetics as well as function from a very young age. The lateral incisors act as a guide in the normal eruption of the permanent canine and absence of upper lateral incisors may lead to canine impaction. The persistence of a primary lateral incisor in the arch beyond the expected time of eruption of its successor often suggests agenesis.

Bilateral missing upper lateral incisors are more common than unilateral ones. Unilateral agenesis is often associated with the microdontia of the corresponding contralateral tooth, which in turn requires higher biomechanical claims in orthodontic therapy
${ }^{1-5}$ Department of Orthodontics and Dentofacial Orthopedics, Kalinga Institute of Dental Sciences, KIIT Deemed to be University, Bhubaneswar, Odisha, India

Corresponding Author: Nivedita Sahoo, Department of Orthodontics and Dentofacial Orthopedics, Kalinga Institute of Dental Sciences, KIIT Deemed to be University, Bhubaneswar, Odisha, India, Phone: +91 7894391909, e-mail: drniveditasahoo81@gmail.com

How to cite this article: Sahoo N, Reddy R, et al. Comparison of Frequency of Congenitally Missing Upper Lateral Incisors among Skeletal Class I, II and III Malocclusions. J Contemp Dent Pract 2019;20(9):1019-1023.

Source of support: Nil

Conflict of interest: None

or often leads to extraction of the hypoplastic tooth to prevent any asymmetric effects as well as a midline shift. ${ }^{6-8}$

The definitive diagnosis requires a mandatory X-ray examination in order to confirm the assumed diagnosis. After clinical and radiological proof, a decision regarding the treatment options must be made, whether it should be treated by orthodontic space closure or by space opening and implantation. Individual evaluation of the treatment choices requires a multidisciplinary approach to achieve the best possible result for the patient. The treatment depends on a number of factors such as facial, occlusal, functional, and periodontal features, as well as individual long-term stability. ${ }^{7-10}$

(c) The Author(s). 2019 Open Access This article is distributed under the terms of the Creative Commons Attribution 4.0 International License (https://creativecommons. org/licenses/by-nc/4.0/), which permits unrestricted use, distribution, and non-commercial reproduction in any medium, provided you give appropriate credit to the original author(s) and the source, provide a link to the Creative Commons license, and indicate if changes were made. The Creative Commons Public Domain Dedication waiver (http://creativecommons.org/publicdomain/zero/1.0/) applies to the data made available in this article, unless otherwise stated. 
The demand for orthodontic treatment in cases of missing upper lateral incisors is high because of the obvious impact that this condition has on both dental and facial esthetics. This is a challenging situation that every orthodontist will encounter on a regular basis.

The aim of the present investigation was to determine the frequency of congenitally missing upper lateral incisors among skeletal class I, II, and III malocclusions and to find out its variability among different genders.

\section{Materials and Methods}

A retrospective study was carried out between January 2017 and December 2017. Orthopantomograms (OPGs) and a lateral cephalogram record search of all of the orthodontic Odia patients aged 12-18 years were taken, which were available in the Kalinga Institute of Dental Sciences, KIIT, Bhubaneswar, Odisha. This retrospective study excluded ambiguous OPGs and a lateral cephalogram of subjects with no proper birth record and distorted images. Ethical Clearance was obtained from institutional ethics committee (IEC approval no. KIMS/KIIT/IEC/135/2017).

\section{Inclusion Criteria}

Were set as per the following criteria:

- Odia ancestry.

- No past record of maxillary lateral incisor extraction.

- No enameloplasty or prosthesis of the maxillary lateral incisors.

- No history of previous orthodontic treatment.

\section{Exclusion Criteria}

- Cleft lip and palate patients.

- Any other craniofacial syndromic patients.

On the basis of the inclusion and exclusion criteria, 1,000 OPGs and a lateral cephalogram were selected. Demographic variables such as gender and age were determined, as shown in Figures 1 to 3 .

Of the selected samples, 290 were males and 710 were females. Orthopantomograms helped diagnose the presence of unilateral/ bilateral maxillary lateral incisors. ANB angle was calculated from the lateral cephalogram in order to divide the subjects into various skeletal malocclusions. Cranio-facial syndromic patients were

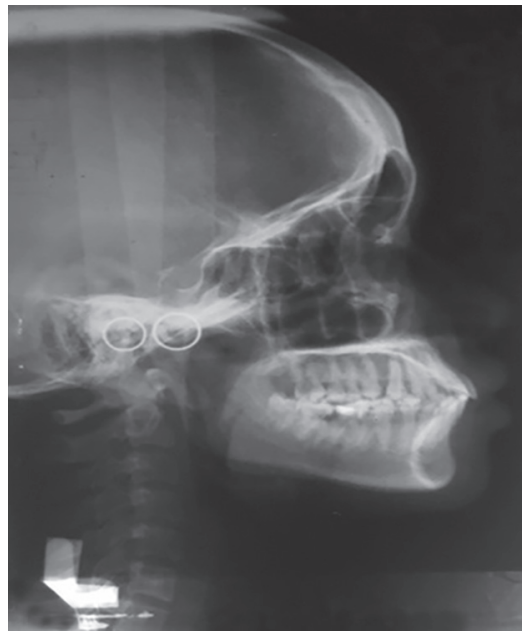

Fig. 1: A lateral cephalogram showing the missing upper left lateral incisor excluded from the study. The OPGs with evidence of impacted maxillary lateral incisors were also not included in the present investigation.

\section{Results and Statistics}

In the sample of 1,000 patients that were chosen from the retrospective survey, 710 (71\%) were female and 290 (29\%) were male patients, as shown in Table 1. A total of 37 (3.77\%) patients were found to have missing maxillary lateral incisors. Of the 37 patients with missing lateral incisors, 28 were females and 9 were males, as shown in Table 2 . Totally, $4.04 \%$ of the female patients and $3.10 \%$ of the male patients were having missing upper lateral incisors in relation to the upper jaw. The frequency of missing upper laterals among male subjects was $0.9 \%$. Totally, $2.8 \%$ of the female patients were having missing maxillary lateral incisors.

On the basis of the gender, $62.16 \%$ had a bilateral expression of missing upper laterals, where $78.26 \%$ were females and $21.73 \%$ were males. An estimated $16.21 \%$ had a left unilateral expression, where $83.33 \%$ were females and $16.66 \%$ were males. A total of $21.62 \%$ had a right unilateral expression, where $62.5 \%$ were females and $37.5 \%$ were males, as given in Table 3.

Skeletal class I malocclusion has a prevalence of $54.16 \%$ of bilaterally missing lateral incisors in the upper arch when compared to $40 \%$ of the left unilateral expression and $37.5 \%$ of the right unilateral expression. In contrast, in skeletal class II malocclusion, the incidence of the right unilateral expression was $37.5 \%$ when compared to the bilateral expression which was $33.33 \%$. The left unilateral expression in skeletal class II malocclusion was only $20 \%$. The highest prevalence of missing laterals in skeletal class III malocclusion was a left unilateral expression, which was around $40 \%$. The incidence of a right unilateral expression was $25 \%$, whereas bilateral absence of upper laterals in skeletal class III malocclusion was $12.5 \%$, as shown in Table 4 .

The inference of the present study indicates that the frequency of missing upper lateral incisor (bilateral) in skeletal class I was found

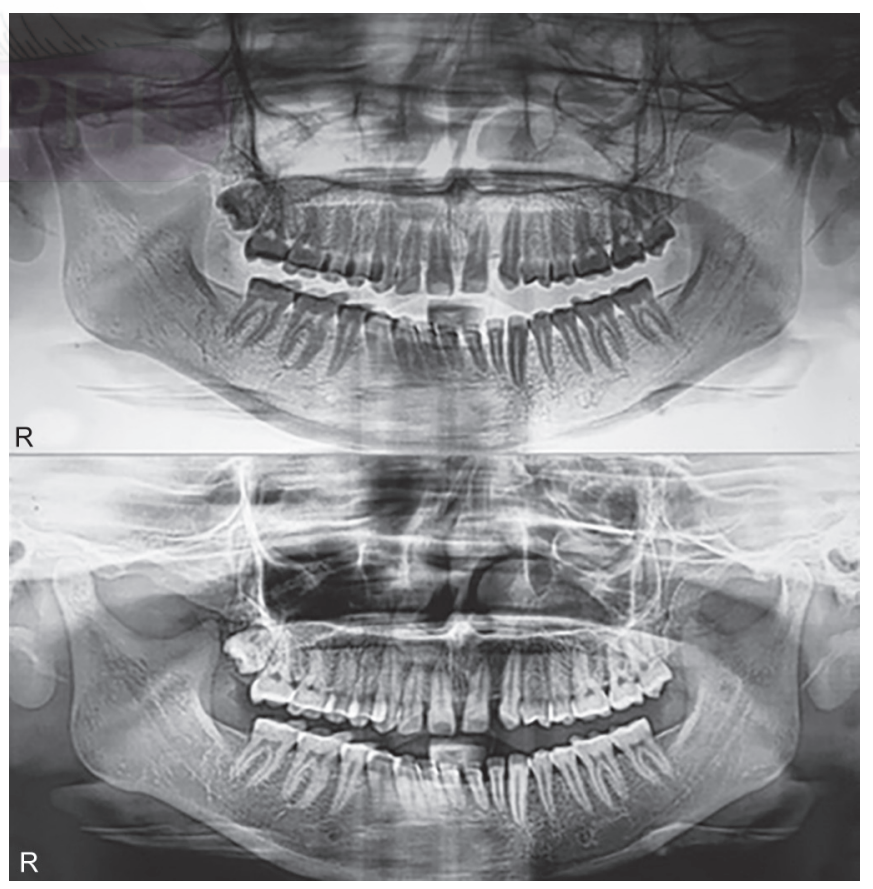

Fig. 2: OPG showing the missing upper left lateral incisor 


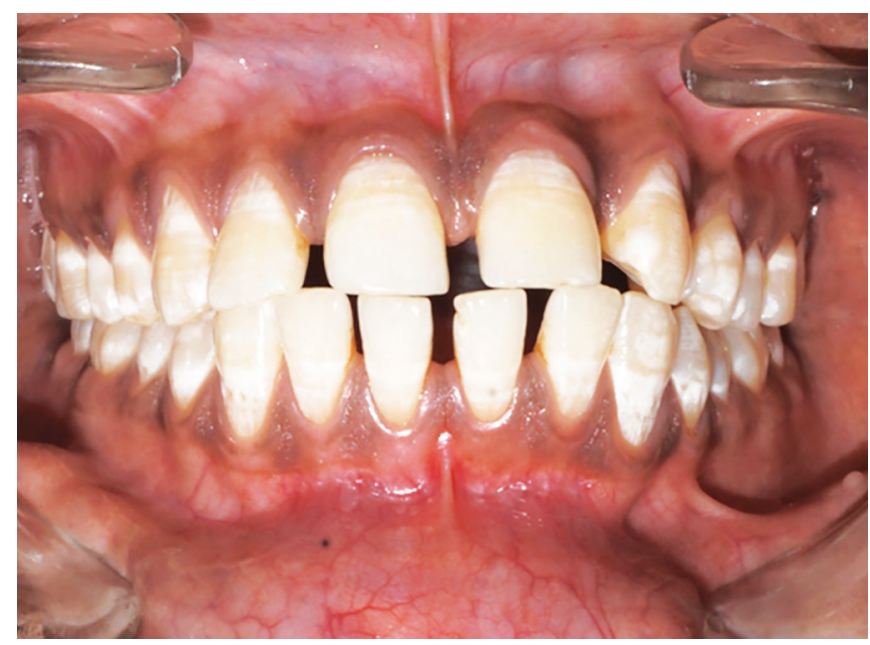

Fig. 3: Image of anteriors in occlusion showing the missing upper left lateral incisor

Table 1: Gender distribution (number of patients treated)

\begin{tabular}{lll}
\hline Gender & Male & Female \\
\hline Number of patients & $290 / 1,000$ & $710 / 1,000$ \\
Frequency & $29 \%$ & $71 \%$ \\
\hline
\end{tabular}

Table 2: Prevalence of missing upper lateral incisor among orthodontic Odia adoloscent population

\begin{tabular}{lll}
\hline Gender & Male & Female \\
\hline $\begin{array}{l}\text { No. of patients with } \\
\text { missing lateral incisor }\end{array}$ & $9 / 1,000$ & $28 / 1,000$ \\
Frequency & $0.9 \%$ & $2.8 \%$ \\
\hline
\end{tabular}

to be $54.16 \%, 33.33 \%$ in skeletal class II and $12.5 \%$ in skeletal class III. Though the results were not of statistical significance, it holds paramount importance in the clinical scenario.

\section{Discussion}

Retrospective data reveal no study being carried out to compare the frequency of congenitally missing upper lateral incisors among Skeletal class I, II, and III malocclusions in an Odia adolescent population. In the present study, the sample size was large in a sequence to acquire epidemiological and clinical information correlated to the non-existence of lateral incisors in upper arch. The utilization of OPGs permitted entry to a supply of dependable, widespread, and effortlessly available data.

The prevalence of congenital missing permanent lateral incisors in the upper arch varies significantly amongst studies. ${ }^{11-16}$ It is essential to have significant epidemiological data on various classes of malocclusion, sequentially to calculate approximately the overall time requirement for correction and management.

The samples that were considered in the present study were selected to achieve a comprehensible and suitable representation of the prevalence pattern of agenesis of the upper permanent maxillary lateral incisor in the orthodontic Odia adolescent population in Bhubaneswar, Odisha, India.

Researchers of other populations also reported a different prevalence pattern of agenesis of maxillary lateral incisors, leading to a conclusion that variations are present between different races. The reasoning to the above differences might be attributed to a racial and ethnic origin.

An investigation was done by Sofaer et al. ${ }^{17}$ in 1971 on a sample of 17,000 high school students from Hawaii. The sample ranged from a full complement of teeth to a congenitally missing maxillary upper lateral incisor. The clinical examination of the patient was done and it was cross checked with radiographs. Generally it was observed

Table 3: Prevalence and statistical significance of missing upper laterals in both genders

\begin{tabular}{|c|c|c|c|c|c|c|c|c|}
\hline \multirow[b]{2}{*}{ Gender } & \multicolumn{2}{|c|}{ Both } & \multicolumn{2}{|c|}{ Left } & \multicolumn{2}{|c|}{ Right } & \multicolumn{2}{|c|}{ Total } \\
\hline & $n$ & $\%$ & $n$ & $\%$ & $n$ & $\%$ & $n$ & $\%$ \\
\hline Female & 18 & 78.26 & 5 & 83.33 & 5 & 62.5 & 28 & 100 \\
\hline$p$ value & $(0.02)$ & & $(0.05)$ & & (0.18) & & $(0.00)$ & \\
\hline Male & 5 & 21.73 & 1 & 16.66 & 3 & 37.5 & 9 & 100 \\
\hline$p$ value & $(0.06)$ & & $(0.14)$ & & $(0.57)$ & & $(0.00)$ & \\
\hline Total & 23 & 62.16 & 6 & 16.21 & 8 & 21.62 & 37 & 100 \\
\hline
\end{tabular}

$p<0.05$ statistical significance

Table 4: Prevalence and statistical significance of missing upper laterals in different skeletal malocclusions

\begin{tabular}{|c|c|c|c|c|c|c|c|c|}
\hline \multirow[b]{2}{*}{ Skeletal class } & \multicolumn{2}{|c|}{ Both } & \multicolumn{2}{|c|}{ Left } & \multicolumn{2}{|c|}{ Right } & \multicolumn{2}{|c|}{ Total } \\
\hline & $n$ & $\%$ & $n$ & $\%$ & $n$ & $\%$ & $n$ & $\%$ \\
\hline I & 13 & 54.16 & 2 & 40 & 3 & 37.5 & 18 & 100 \\
\hline$p$ value & \multicolumn{2}{|c|}{$(0.15)$} & \multicolumn{2}{|c|}{$(0.08)$} & \multicolumn{2}{|c|}{$(0.20)$} & \multicolumn{2}{|c|}{$(0.00)$} \\
\hline II & 8 & 33.33 & 1 & 20 & 3 & 37.5 & 12 & 100 \\
\hline$p$ value & \multicolumn{2}{|c|}{$(0.01)$} & \multicolumn{2}{|c|}{$(0.24)$} & \multicolumn{2}{|c|}{$(0.06)$} & \multicolumn{2}{|c|}{$(0.00)$} \\
\hline III & 3 & 12.5 & 2 & 40 & 2 & 25 & 7 & 100 \\
\hline$p$ value & \multicolumn{2}{|c|}{$(0.52)$} & \multicolumn{2}{|c|}{ (1.17) } & \multicolumn{2}{|c|}{$(0.16)$} & \multicolumn{2}{|c|}{$(0.00)$} \\
\hline Total & 24 & 64.86 & 5 & 13.51 & 8 & 21.62 & 37 & 100 \\
\hline
\end{tabular}

$p<0.05$ statistical significance 
that in cases where there was a missing lateral incisor, the size of the normal central incisor was comparatively larger.

A radiographic study was conducted by Le Bot et al., ${ }^{18}$ where a sample size of 200 males from the French population with maxillary lateral incisor agenesis was considered for checking the prevalence of missing third molars. The authors concluded that $39.6 \%$ of the study samples who reported with missing teeth had an agenesis of third molar in comparison to the control group, with a prevalence of $12.4 \%$ in the control group.

Review of literature reveals tooth agenesis being related to some tooth conditions such as supernumerary tooth, retained deciduous tooth and ectopic eruption, microdontia or peg-shaped incisors, taurodontism, and transposition. Conversely, the linkage between dental anomalies and missing MLI has been sparsely documented in the database.

A study done by Aphraim and Shubha ${ }^{19}$ stressed the importance of early diagnosis of missing lateral incisors in the mixed dentition period, its consequences, and the potential earliest management during this period.

In the present investigation, a prevalence of agenesis of missing lateral incisors as high as $3.77 \%$ was documented. In the present study, a striking increase in percentage of $\mathrm{MLI}$ agenesis was found in females. ${ }^{19}$ An estimated $2.8 \%$ of the females had missing upper laterals when compared to males with a prevalence of $0.9 \%$.

A similar study was done by Horowitz ${ }^{11}$ in a sample of 1,000 subjects of the Portuguese population. He found an incidence of $1.11 \%$ of missing lateral incisors. Another study was conducted by Aasheim and Ogaard ${ }^{21}$ in a Nordic sample being screened for orthodontic examination. The authors revealed that the frequency of missing upper laterals was around $2 \%$, which was similar to our study. Kabbani et al. ${ }^{22}$ evaluated the prevalence of a congenital absence of maxillary lateral incisors in 8,000 school children with an equal number of males and females (age range: 12-15 years) in a Syrian population. The authors concluded that prevalence of isolated maxillary lateral incisors agenesis was $1.15 \%$. Some studies have recorded an overall prevalence of congenitally missing teeth in a range from $2 \%$ to $16.3 \% .^{23}$

The variability in results amongst different population might be due to genetics or environmental influences.

The teeth that most commonly fail to erupt are those that erupt in vital terminal areas of the dental lamina. The most commonly impacted tooth are the upper lateral incisor, second premolars, and third molars.

There is a close connection between agenesis of lateral incisors in the upper arch and second premolars. At molecular level, some factors affecting neural structure formation might have an influence on tooth formation. This can lead to some defects in molecular factors, which influence neural growth, leading to failure of tooth formation. The mutations in genes responsible for tooth development are marked as PAX9, MSX1, and AXIN2. Among these, MSX1 is usually related to congenitally missing third molars, second premolars, maxillary first premolar, and incisors.

The agenesis of laterals in upper arch is frequently seen in females when compared to males. ${ }^{11,13,14,24,25}$ The findings of our study also supports this literature. The explanation for the above finding could be attributed to variation in sexes owing to their differences in tooth eruption and skeletal growth. ${ }^{20}$

During the primary stages of development, the space that is available for the lateral incisor will depend upon the space that is left out after development of the centrals and canines. There is a competition of the lateral incisor with its neighbor for space, as the canine and central incisors develop prior to the lateral incisor. The tooth size asymmetry as stated by Sofaer ${ }^{17}$ might be due to some environmental disturbances or deprived primordium or both.

Of the samples with missing upper laterals, $62.16 \%$ of the samples had a bilateral expression, $16.21 \%$ had a left unilateral expression and $21.62 \%$ had a right unilateral expression.

Though the results of the present study do not have much statistical significance as indicated by the results, it has immense clinical importance. It is very important on the part of the clinician to diagnose the type of missing laterals either unilateral or bilateral, check for the type of skeletal bases and the gender, and accordingly plan the treatment. ${ }^{26-29}$

Totally, 2,662 dental patients in Palestine were evaluated for the prevalence of missing lateral incisors. The percentage was found to be $1.91 \%$. Unilateral agenesis is accounted for $66.6 \%$ of the total cases. Around $79 \%$ of the unilateral cases were on the left side, while $21 \%$ were on the right side. Bilateral agenesis accounted for $33.3 \%$ of the total cases. ${ }^{30}$

Isolated bilateral absence of maxillary lateral incisors is associated with decreased mesio-distal widths in both maxillary and mandibular anterior segments. ${ }^{31,32}$

The management of maxillary lateral incisors can be done either by orthodontic space closure of canine mesial positioning and reshaping or by a prosthodontic intervention. ${ }^{33,34}$ Osseointegrated implants can be used to replace congenitally missing upper lateral incisors. ${ }^{35-38}$ Customised lingual orthodontic appliances and the adjunctive use of direct skeletal anchorage derived from two palatal mini-implants was used in a patient to close the space of a congenitally absent lateral incisor. ${ }^{39}$

The strength of our study is that there is surprisingly little information in the literature on the prevalence of missing maxillary lateral incisors and the skeletal pattern in an orthodontic population. Orthodontic patients do not necessarily replicate the number of individuals in the population with tooth agenesis; this will be dependent on the availability of orthodontic treatment and its uptake in this particular population. However, retrospective studies rely on good record keeping and orthodontic patients often have more complete records. Review of literature suggests that orthodontic space closure in missing laterals produced stable results and were accepted better by patients compared to prosthetic rehabilitation. ${ }^{40}$

The limitation of our study was that it was retrospective and radiographic in nature. In future, a prospective clinical-radiographic study with a large number of samples may be considered.

Knowledge of the pattern and prevalence of tooth agenesis is important for treatment planning. If done properly and timely, an interdisciplinary treatment might prevent the patient from esthetic and functional discrepancies that may interfere with adequate development and growth, which may cause functional, occlusal, and esthetic disharmony.

This study provides useful information and statistics regarding lateral incisor agenesis and helps learn more about the prevalence of maxillary lateral incisor agenesis. The authors emphasize the importance of early diagnosis and referral to reduce or prevent a number of complications that may affect function and esthetics. ${ }^{30}$

\section{Conclusion}

The prevalence rate of congenitally missing upper lateral incisors in orthodontic adolescent Odia population in Bhubaneswar is $3.77 \%$. 
The females had a greater percentage of agenesis of the upper lateral incisor (2.8\%) when compared to males (0.9\%). This difference might be due to genetic variations or different environmental conditions. A multidisciplinary treatment protocol must be planned to manage missing maxillary lateral incisors and to restore the impaired esthetics and function.

\section{Clinical Significance}

The comparison of frequency of congenitally frequency of missing upper lateral incisors among skeletal class I, II, and III malocclusions is vital for diagnosis and treatment planning. A multidisciplinary treatment protocol must be planned to manage missing the maxillary lateral incisor and to restore the impaired esthetics and function.

\section{References}

1. Kokich VO Jr, Kinzer GA. Managing congenitally missing lateral incisors Part II: Tooth-supported restorations. J Esthet Restor Dent 2005;17(2):76-84. DOI: 10.1111/j.1708-8240.2005.tb00089.x.

2. Woolf CM. Missing maxillary lateral incisors: a genetic study. Am J Hum Genet 1971;23(3):289-296.

3. Fekonja A. Hypodontia in orthodontically treated children. Eur J Orthod 2005;27:457-460. DOI: 10.1093/ejo/cji027.

4. Ben-Bassat $Y$, Brin I. Skeletal and dental patterns in patients with severe congenital absence of teeth. Am J Orthod Dentofacial Orthop 2009;135(3):349-356. DOI: 10.1016/j.ajodo.2008.09.002.

5. Witkop C. Agenesis of succedanceous teeth: an expression of the homozygous state of the gene for the pegged or missing maxillary lateral incisor trait. Am J Med Genet 1987;26:431-436. DOI: 10.1002/ ajmg.1320260222.

6. Arte $S$, Nieminen $P$, et al. Characteristics of incisor-premolar hypodontia in families. J Dent Res 2001;80:1445-1450. DOI: $10.1177 / 00220345010800051201$.

7. Nieminen P. Genetic basis of tooth agenesis. J Exp Zool Part B Mol Dev Evol 2009;312:320-342. DOI: 10.1002/jez.b.21277.

8. DeCoster PJ, Marks LA, et al. Dentalagenesis: genetic and clinical perspectives. J Oral Pathol Med 2009;38:1-17. DOI: 10.1111/j.16000714.2008.00699.x.

9. Kapadia $H$, Mues $G$, et al. Genes affecting tooth morphogenesis. Orthod Craniofac Res 2007;10:105-113. DOI: 10.1111/j.16016343.2007.00395.x.

10. Moyers RE. Early Treatment, Handbook of Orthodontics, 4th ed.; 1988.

11. Horowitz JM. Aplasia and malocclusion: a survey and appraisal. Am J Orthod 1966;52:440-453. DOI: 10.1016/0002-9416(66)90122-9.

12. Baccetti T. A controlled study of associated dental anomalies. Angle Orthod 1998;68:267-274.

13. Helm S. Malocclusion in Danish children with adolescent dentition: an epidemiologic study. Am J Orthod 1968;54:352-366. DOI: 10.1016/0002-9416(68)90304-7.

14. Laskaris G. Color atlas of oral diseases in children and adolescents. Thieme, New York Leitao P 1993. Prevalencia da ma oclusaoemcrianças de 12 anos da cidade de Lisboa. Parte I. Revista Portuguesa de Estomatologia e Cirurgia Maxilofacial 2000;33:193-201.

15. Moyers R. Handbook of Orthodontics. Year Book Medical Publishers; 1988.

16. Rolling S. Hypodontia of permanent teeth in Danish school children. Scand J Dent Res 1980;88:365-369.

17. Sofaer JA, Chung CS, et al. Developmental interaction, size and agenesis among permanent maxillary incisors. Hum Biol 1971;43: 36-45.

18. Le Bot P, Salmon D. Congenital defects of the upper lateral incisors (ULI): condition and measurements of the other teeth, measurements of the superior arch, head and face. Am J Phys Anthrop 1977;46: 231-244. DOI: 10.1002/ajpa.1330460204.

19. Aphraim R, Shubha M. Agenesis of maxillary primary and permanent lateral incisor. Int J Sci Eng Res 2014;5(9):67-69.

20. Proffit WR, Fields HW, et al. Contemporary Orthodontics. St. Louis, MO: Mosby Elsevier; 2007.

21. Aasheim B, Ogaard B. Hypodontia in 9-year-old Norwegians related to need of orthodontic treatment. Scand J Dent Res 1993;101:257-260. DOI: 10.1111/j.1600-0722.1993.tb01115.x.

22. Kabbani $\mathrm{T}$, Abdullah NM, et al. Prevalence of isolated maxillary lateral incisor agenesis in Syrian adolescents. J Orofac Orthop 2016; 1-8.

23. Srivathsa SH. Congenitally missing maxillary central incisor or solitary median maxillary central incisor? Int J Orofac Res 2018;3:17-19.

24. Johannsdottir B, Wisth PJ, et al. Prevalence of malocclusion in 6-yearold Icelandic children. Acta Odontol Scand 1997;55:398-402. DOI: 10.3109/00016359709059206.

25. Magnusson TE. Prevalence of hypodontia and malformations of permanent teeth in Iceland. Community Dent Oral Epidemiol 1977;5:173-178. DOI: 10.1111/j.1600-0528.1977.tb01635.x.

26. Svinhufvud E, Myllarniemi S, et al. Dominant inheritance of tooth malposition and their association to hipodontia. Clin Genet 1988;34:373-381.

27. Sejrsen B, Kjær l, et al. Agenesis of permanent incisors in a mediaeval maxilla and mandible: aetiological aspects. Eur J Oral Sci 1995;103: 65-69. DOI: 10.1111/j.1600-0722.1995.tb00118.x.

28. Thesleff I. Two genes for missing teeth. Nat Genet 1996;13:379-380. DOI: 10.1038/ng0896-379.

29. Kjær I. Can the location of tooth agenesis and the location of initial bone loss seen in juvenile periodontitis be explained by neural developmental fields in the jaws? Acta Odontol Scand 1997;55:70-72. DOI: 10.3109/00016359709091945.

30. Arandi NZ, Mustafa S. Maxillary lateral incisor agenesis; a retrospective cross-sectional study. Saudi Dent J 2018;30(2):155-160. DOI: 10.1016/ j.sdentj.2017.12.006.

31. Yakoob O, DiBiase AT, et al. Relationship between bilateral congenital absence of maxillary lateral incisors and anterior tooth width. Am J Orthod Dentofacial Orthop 2011;139:229-233. DOI: 10.1016/ j.ajodo.2010.04.030.

32. Caterini L, Mezio M, et al. Clinical features of maxillary lateral incisor agenesis and associated dental anomalies: a systematic review. Webmed Central Orthod 2017;8(10):WMC005341.

33. Kiliaridis S, Sigira M, et al. Treatment options for congenitally missing lateral incisors. Eur J Oral Implantol 2016;9(suppl 1):S5-S24.

34. Ambekar DA, Kangane DS, et al. Management of Congenital Missing Unilateral Maxillary Lateral Incisor Treated with Begg'sMechanotherapy: a Case Report. IOSR-JDMS 2015;14(3):7-10.

35. Muhamad AH, Azzaldeen A, et al. Esthetic management of congenitally missing lateral incisors with single tooth implants: a case study. International. J Curr Res 2015;7(04):14600-14606.

36. Tepper G, Killaridis S, et al. Foundation for oral rehabilitation consensus text on "the rehabilitation of missing single teeth. Eur J Oral Implantol 2016;9(1):173-178.

37. Garg A, Garg M, et al. A comprehensive orthodontic, surgical, and prosthodontic rehabilitation of congenital unilateral cleft lip and cleft palate patient: An interdisciplinary case report. J Indian Orthod Soc 2018;52(S1):40-48. DOI: 10.1177/0974909820180507S.

38. Haryani J, Singh GP, et al. Orthodontic Space Closure for Management of Congenitally Missing Upper Lateral Incisors. J Clin Orthod 2017;51(4):223-228.

39. Pies S, Wilmes B, et al. Congenitally absent maxillary lateral incisors. a case report illustrating the use of a Mesialslider and a customised lingual appliance. Aust Orthod J 2018;34:103-116.

40. Robertsson S, Mohlin B. The congenitally missing upper lateral incisor. a retrospective study of orthodontic space closure versus restorative treatment. Eur J Orthod 2000;22:697-710. DOI: 10.1093/ejo/22.6.697. 